\title{
Heart transplantation: new realities, challenges and developments - surgical perspectives
}

\begin{abstract}
In this second article we will discuss the important surgical developments that are changing the realities on the ground of heart transplantation. These developments have arisen as a result of the increasing threats to heart transplantation, principally, the paucity of donors. The emergence of mechanical circulatory support as a bridge to transplantation or as destination therapy will be discussed as well as the possibility of bridging these patients in rare instances to a recovery from heart failure. Advances in organ preservation will be discussed as well as ex vivo perfusion and the possibility of retrieval from circulation arrested donors. Finally, advances in surgical technique will be discussed.
\end{abstract}

Keywords: heart failure, transplantation, mechanical circulatory support, total artificial heart, immunosuppression
Volume I Issue 3 - 2014

\author{
Babar B Chaudhri \\ Department of Cardiovascular, Thoracic Surgery and \\ Intrathoracic Transplantation, Sir HN Reliance Foundation \\ Hospital, India
}

\begin{abstract}
Correspondence: Babar B Chaudhri, Department of Cardiovascular, Thoracic Surgery and Intrathoracic Transplantation, Sir HN Reliance Foundation Hospital, Raja Ram Mohan Roy Marg, Mumbai, 40004, India, Tel 91 7738164236, Email bchaudhri@mac.com
\end{abstract}

Received: May 07, 2014 | Published: June 27, 2014
Abbreviations: LVAD, left ventricular assist devices; INTERMACS, interagency registry for mechanically assisted circulatory support; MCS, mechanical circulatory support; NYHA, New York heart association; TAH, total artificial heart

\section{Mechanical circulatory assist devices}

In recent years, the use of MCS devices in treating patients with end-stage heart disease has increased significantly, as a bridge to transplantation and as destination therapy for transplant ineligible candidates. This increase is based on the accumulated experience with new second-generation continuous-flow devices which show significant improvements in survival, functional capacity and quality of life (Figure 1). 1,2 On the basis of the Heart Mate II Registry experience (1300 patients); guidelines for the clinical management of patients treated with continuous-flow devices have been published. ${ }^{3}$ Risk scoring systems, such as the Seattle Heart Failure Model $^{4}$ and the Cumulative Risk Score for 90-Day in-Hospital Mortality ${ }^{5}$ and the Destination Therapy Risk Score have been investigated to stratify patients who might benefit from LVAD support. ${ }^{6}$ The Interagency Registry for Mechanically Assisted Circulatory Support (INTERMACS) registry, follows all long-term MCS systems in the United States shows I year survival for continuous flow LVAD IS $82.2 \%$.

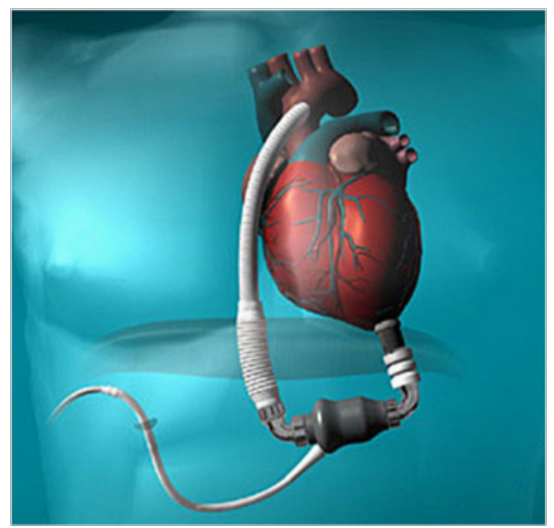

Figure I Theoretic Heartmate II LVAD, schematic of device in situ.
Right ventricle failure is a leading cause of morbidity and death after LVAD implant with an incidence of about $35 \%$, and can be very difficult to predict. ${ }^{7,8}$ Various methods to assess right ventricle function pre- and postoperatively have been developed. ${ }^{2}$ Right ventricular failure risk scores have been created that stratify the risk of right ventricular failure (RVFRS) and death after LVAD implantation. One such RVFRS found independent predictors of right ventricular failure to include vasopressor requirement, aspartate aminotransferase $>80 \mathrm{IU} / \mathrm{L}$, bilirubin $>2.0 \mathrm{mg} / \mathrm{dL}$ and creatinine $>2.3 \mathrm{mg} / \mathrm{dL} .^{7}$ Another study developed a score to predict RVAD need after LVAD placement, which included factors of cardiac index, right ventricular stroke work index, severe preoperative right ventricular dysfunction, creatinine, previous cardiac surgery and systolic blood pressure ${ }^{8}$ More recently the presence of severe TR and a tricuspid annulus of $>43 \mathrm{~mm}$ and right ventricular sphericity have been proposed as predictive of occult RV failure and need for biventricular support. Reduced survival with RV failure has been shown even when LVAD support is supplemented with RVAD implantation (Figure 2).

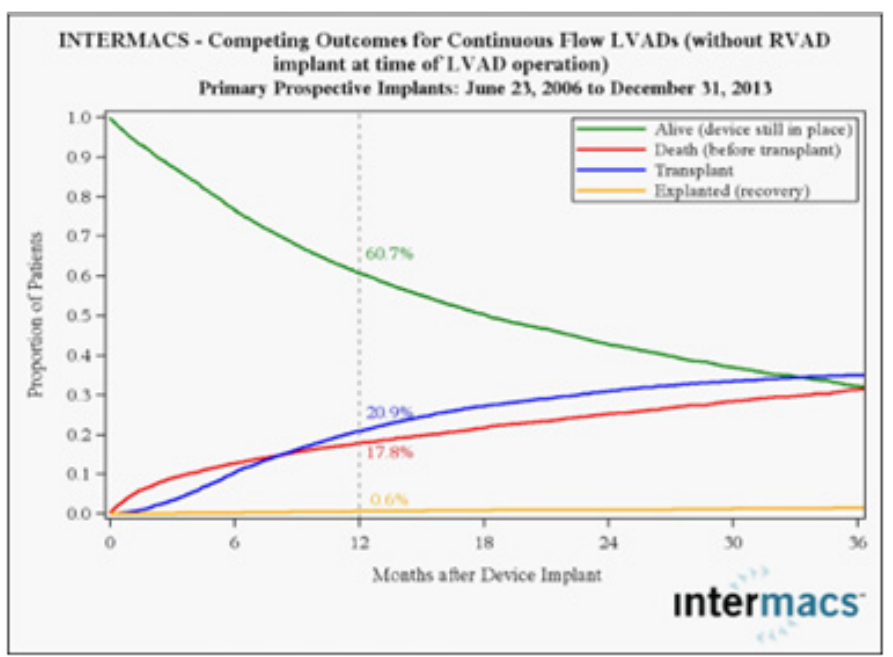

Figure 2 INTERMACS patient profiles and timeframe for initiating mechanical circulatory support with LVAD only (with permission from INTERMACS Quarterly Statistical Report 2013, 4th Quarter, Implant and event dates: June 23, 2006 to December 31, 2013 http://www.uab.edu/medicine/intermacs). 
INTERMACS has defined patient profiles that can help identify risks associated with the timing of implant ${ }^{9}$ (Table 1). In the future, the INTERMACS patient profile would be a useful tool to improve management and outcomes of patients who need VAD implant, and unify criteria for future clinical trials and devices. As more LVAD patients are listed for heart transplant, a competition has occurred for organs between stable LVAD supported registrants and less stable registrants listed status UNOS $1 \mathrm{~A}$ or $1 \mathrm{~B}$. A recent study found that stable LVAD patients had significantly less 30-day risk of events compared to other status $1 \mathrm{~A}$ patients concluding that allowance of 30 days of elective status $1 \mathrm{~A}$ time should not be allocated to stable registrants with implanted LVADs. ${ }^{10}$ As VAD technology improves, further revisions to the allocation system will need to be recommended.
The INTERMACS registry also publishes outcomes for mechanical circulatory support. I year survival for continuous flow LVAD is $82.2 \%$ and $60.3 \%$ where a RVAD is additionally implanted. Temporary MCS systems are available that can be implanted quickly to improve cardiac output in patients with severe acutely decompensated heart failure. The CentriMag ${ }^{11}$, TandemHeart ${ }^{12}$, Impella ${ }^{13}$ and Circulite ${ }^{14}$ VADs offer versatility for use in many patients and in multiple hospital settings. Current clinical trials suggest that treatment of temporary VADs does not necessarily correlate with better survival, but merely comprise a component of treatment leading to recovery, upgrade to fully implantable systems as a bridge to transplant or destination therapy, or transplantation (Table 1). ${ }^{15,16}$

Table I INTERMACS Profile and Description and Timescale to MCS. ${ }^{3}$

\begin{tabular}{|c|c|c|c|}
\hline I & Crashing and burning & Critical cardiogenic shock. & Within hours \\
\hline 2 & Progressive decline & Inotropic dependence with continuing deterioration. & Within a few days \\
\hline 3 & $\begin{array}{l}\text { Stable but inotropic } \\
\text { dependent }\end{array}$ & $\begin{array}{l}\text { Describes clinical stability on mild-to-moderate doses of intravenous inotropes (patients } \\
\text { stable on temporary circulatory support without inotropes are within this profile). }\end{array}$ & Within a few weeks \\
\hline 4 & $\begin{array}{l}\text { Recurrent advanced } \\
\text { heart failure }\end{array}$ & Recurrent" rather than "refractory"decompensation. & Within weeks to months \\
\hline 5 & Exertion intolerant & Describes patients who are comfortable at rest but are exercise intolerant. & Variable \\
\hline 6 & Exertion limited & $\begin{array}{l}\text { Describes a patient who is able to do some mild activity but fatigue results within a few } \\
\text { minutes of any meaningful physical exertion. }\end{array}$ & Variable \\
\hline 7 & Advanced NYHA III & $\begin{array}{l}\text { Describes patients who are clinically stable with a reasonable level of comfortable activity, } \\
\text { despite history of previous decompensation that is not recent. }\end{array}$ & Not a candidate for MCS \\
\hline
\end{tabular}

INTERMACS, Interagency Registry for Mechanically Assisted Circulatory Support; MCS, Mechanical Circulatory Support; NYHA, New York Heart Association

For long-term support, devices are being designed which will be smaller, without externalized drive-lines connecting the device to a console and be more durable. The third generation of blood pumps with magnetically levitated rotors has shown satisfactory 1-year survival. ${ }^{17}$ The smaller size and weight of continuous-flow devices has allowed an extension of the new VADs into smaller patients, especially, women. Clinical trial of fully implantable ventricular assist system with a wireless resonant coupling power source is currently undergoing evaluation in the United States which if successful will greatly reduce the incidence of drive line infections, which is the weakest point of the technology of current fully implantable systems. ${ }^{18}$

Many recent studies have focused on the reversed molecular and cellular alterations, such as improved $\beta$-adrenergic responses and decreased calcium-regulating gene expression, in patients using LVAD as a bridge to recovery therapy. ${ }^{19,20} \mathrm{~A}$ major hallmark of patients with heart failure is abnormal calcium cycling into and out of the sarcoplasmic reticulum of cardiomyocytes. Over expression of SERCA in the presence of high concentrations of $\beta$ agonists have been shown not to increase the incidence of arrhythmias or after-contractions in isolated rabbit myocytes. ${ }^{21,22}$ Multiple studies have revealed that reduced SERCA2a expression and decreased phospholamban phosphorylation both contribute to this impairment by raising the concentration of $\mathrm{Ca}^{2+}$ in the cytosol during diastole and reducing it during systole. ${ }^{23,24}$ Recently, gene therapy in human subjects, targeted on SERCA2a has been proven to be safe with trends toward clinical improvement. ${ }^{25}$ Research that has flowed from lab based SERCA2a over expression has led to a clinical trial, "SERCA2a" which will examine the functional effects of over expression of SERCA2a in a patient population with a fully implantable LVAD and CUPID2 will assess whether gene therapy to increase SERCA2a is safe and can improve quality, length of life, and reduce emergency hospital admissions, for heart failure patients. Functional recovery has been observed in a subset of heart failure patients ${ }^{20,21}$. It is reported that changes in excitation-contraction coupling, instead of regression of cellular hypertrophy, predict clinical recovery. ${ }^{19,20}$

Recently, a clinical trial using clenbuterol ( $\beta-2$ agonist and anabolic agent) and LVAD in refractory non-ischemic heart failure patients, reported recovery of heart function in $60 \%$ of patients $(n=20)$ with non-ischemic cardiomyopathy that allows the pump to be explanted (Harefield Recovery Protocol Study for Patients With Refractory Chronic Heart Failure, HARPS). ${ }^{26}$ It has been reported that LVAD therapy is associated with decreased collagen turnover and cross linking and increased tissue angiotensin II. Furthermore, LVAD combined with angiotensin-converting enzyme inhibition results in decreased tissue angiotensin II and collagen cross-linking, normalizes left ventricular end-diastolic pressure volume relationships and is associated with modestly higher rates of bridge to recovery. ${ }^{27}$ Other adjunctive treatments including other medications, cell or gene therapy with over expression of SERCA2a might in conjunction with VAD support provide a meaningful alternative therapy in patients with severe heart disease (Figure 3$)^{28}$

The total artificial heart (TAH) for circulatory support was designed many years ago and has undergone multiple iterations. It is now, at last, increasingly being used in patients with right heart failure or biventricular failure. ${ }^{29} \mathrm{It}$ is indicated as a bridge to transplant in patients with INTERMACS 1 heart failure who are at risk of imminent death. Scenarios include stone heart after cardiac surgery, cardiovascular failure after global infarction and acute post-infarction VSD. Clinical 
experience with TAH (SyncardiaTM) has suggested that it can serve as an alternative for survival with a reasonable complication rate in appropriate candidates with end stage heart failure (Figure 4).$^{30}$ More stable patients can be effectively managed with fully implantable LVAD systems and has limited the use of the TAH.

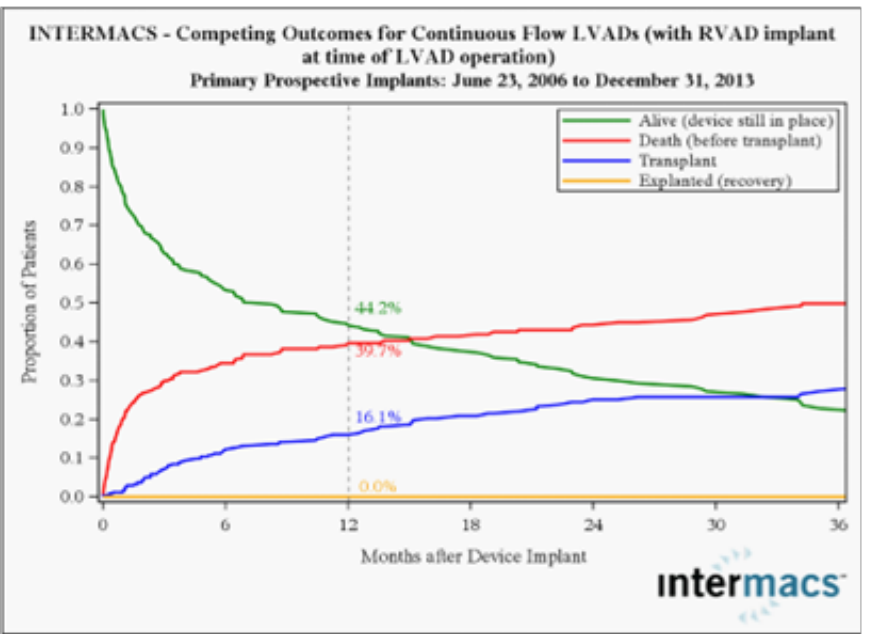

Figure 3 INTERMACS patient profiles and timeframe for initiating mechanical circulatory support with LVAD and concomitant RVAD implantation (with permission from INTERMACS Quarterly Statistical Report 2013, 4th Quarter, Implant and event dates: June 23, 2006 to December 3I, 2013 http://www.uab. edu/medicine/intermacs).

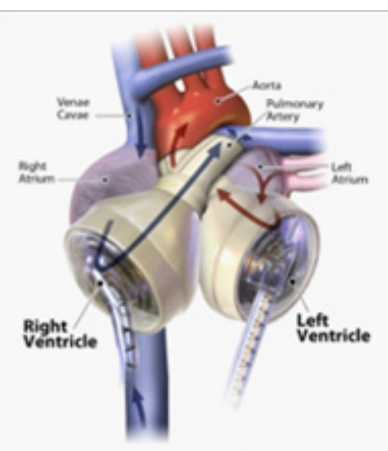

Total Artificial Heart

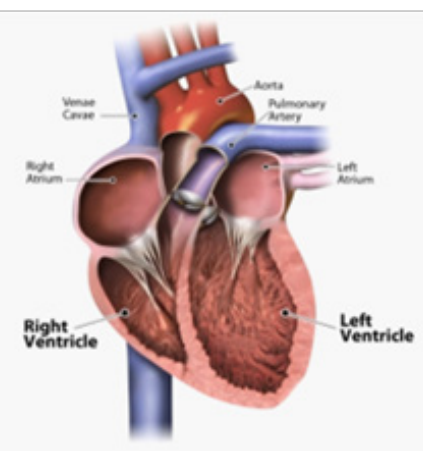

Human Heart
Figure 4 Syncardia $^{\mathrm{TM}}$ Total Artificial Heart, schematic of implant.

\section{Advances in organ preservation}

\section{Heart retrieval}

The retrieval process is a highly organized process as a part of a multidisciplinary, multi-organ retrieval. The assessment takes place with the assistance of a Swan Ganz catheter and occasionally with transesophageal echocardiography to assess function and the presence of valvular disease or other anomalies. After median sternotomy, the pericardium is opened and elevated and the heart is inspected and palpated for size, contractility and anomalies. The coronary arteries are assessed for damage or palpable coronary artery disease. The inferior vena cava (IVC), superior vena cava (SVC) and ascending aorta are dissected. Heparin is administered. The retrieval commences by cross clamping the aorta as high as possible. Cardioplegia solution $(15 \mathrm{ml} / \mathrm{kg})$ is administered via a cannula in the ascending aorta until asystole occurs. The heart is cooled with topical ice-cold saline. The IVC is divided and an incision is made in the left atrial appendage especially if the lung is concurrently retrieved to prevent distension of the LV. After giving cardioplegia, the aorta is divided as high as possible. The pulmonary artery is divided at the level of its bifurcation. The SVC is divided at the level of the azygos vein. If the heart and lungs are to be retrieved, the left atrium is incised at the junction of the left superior pulmonary vein, and extended inferiorly. Care is taken to avoid injury to the coronary sinus. The incision continues inferiorly and then to the junction of the right inferior pulmonary veins and left atrium. By this means the heart is excised with a cuff of left atrium and adequate cuff of left atrium is left continuous with the pulmonary veins to facilitate lung retrieval and subsequent implantation. If the heart alone is to be retrieved, then the pulmonary veins are divided and the intact left atrium is left in continuity with the retrieved heart. Meticulous preservation by administration of adequate cardioplegia and topical cooling is required. The retrieved heart is stored in icecold saline, triple polythene bagged and transported in ice to ensure adequate preservation. It is important to expeditiously transport the heart to the implant centre to ensure a tolerable ischemic time of less than 4 hours. This is the widely used cold static transportation method. There is an inverse relationship between ischemic time and post-transplant survival. ${ }^{31}$

Longer transportation distances and organ retrieval from sites with limited abilities for pre-retrieval evaluation have to be considered. The development of ex vivo perfusion systems such as the Organ Care System ${ }^{\circledR}$ (OCS) (Trans Medics, Andover, MA, USA) (Figure 5) may extend the extracorporeal period by reducing the periods of cold and warm ischemia, with the possibility to constantly evaluate and interact with the retrieved heart during organ transport. The retrieval process is conducted with the administration of cardioplegia, and then the heart is placed on an ex-vivo perfusion system where it is reperfused. ${ }^{32}$ One of the potential advantages of the OCS $^{\circledR}$ is the ability to perform coronary angiography of the donor heart, if a pre-explanations angiography evaluation is not possible at the donor hospital and if significant evidence for coronary artery disease in the donor heart becomes known, because of the donor's medical history or after palpation of sclerotic coronary ostia. With the donor heart is still in the OCS, ${ }^{\circledR}$ not only is it possible to measure metabolic parameters and pressures, but even coronary angiography is feasible. With the increasing international demand for donor organs, such ex vivo examinations might play a more important role, because longer transportation distances can be accepted and organs from suboptimal donors without pre-explanations diagnostics may be considered at donor sites with limited diagnostic options. Current organ retrieval in the US is limited to around 500 nautical miles. Ex vivo perfusion systems allow extended transport time and distances. This could allow national retrieval networks in India and China. If proven effective, this technology may decrease early graft failure and allow increased utilization of available organs. Its potential to decrease ischemic time may also give greater opportunity for prospective cross-matching in heart transplantation. ${ }^{33}$

\section{Retrieval from circulation arrested donors}

Another recent threat to cardiac transplant is the increased usage of circulation deceased donors (DCD). In the UK in particular this has led to a decline in the availability of brain stem dead donors (DBD). Cardiac transplantation in other health systems is still hindered by the lack of donor organs. The first cardiac transplants done used hearts retrieved from circulation deceased donors (DCDs). More recently, a cohort of infant hearts were retrieved and successfully transplanted. Concerns on the ethics of the hands off time, which were less than the recommended 10-15minutes once cardiac arrest had taken place, has led to this practice being halted for reconsideration. ${ }^{34}$ 


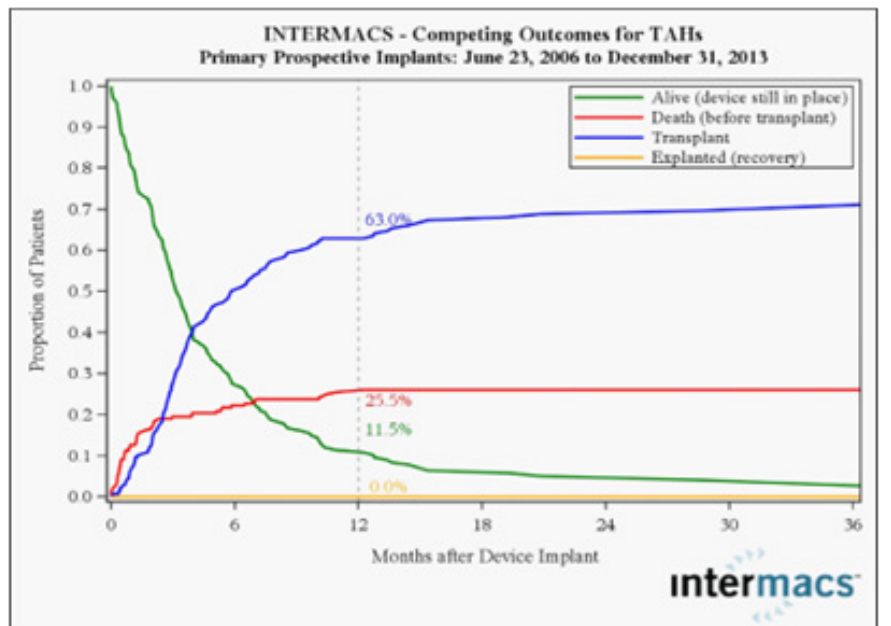

Figure 5 INTERMACS patient profiles and timeframe for Total Artificial Heart (with permission from INTERMACS Quarterly Statistical Report 2013, $4^{\text {th }}$ Quarter, Implant and event dates: June 23, 2006 to December 31, 2013 http://www.uab.edu/medicine/intermacs).

The impact of donor cardiac arrest on orthotropic heart transplantation was investigated using the United Network for Organ Sharing database (UNOS). In 856 out of a total of 19980 cases, the donors from the UNOS series, had histories of cardiac arrest, and in the remainder, there was no history of donor cardiac arrest. The unadjusted 1-, 5-, and 10-year actuarial survival rates between the arrest and the non-arrest groups were not significantly different. Multivariate logistic regression demonstrated no difference in survival in the donor arrest group at 30days, 1year, or 3years. Furthermore, the adjusted Cox proportional hazard model for cumulative survival also showed no survival difference between the 2 groups. If standard recipient and donor transplantation criteria are met, a history of donor cardiac arrest should not prohibit the potential consideration of an organ for transplantation. ${ }^{35}$

A severe donor organ shortage leads to the death of a substantial number of patients who are listed for transplantation. The use of hearts from donors after circulatory death could significantly expand the donor organ pool, but due to concerns about their viability, these are currently not used for transplantation. Short-term extra corporeal circulation with in situ normo-thermic machine perfusion (MP) may allow re-animation and resuscitation of the previously arrested heart in DCD donors. This has been demonstrated in rat and porcine models. Porcine DCD hearts after $29 \mathrm{~min}$ of warm ischemia can be reanimated using in situ extracorporeal circulation. ${ }^{36}$

For DCD hearts, a strategy of pre-reperfusion cardioplegia, followed by continuous warm blood perfusion, is superior to cold storage. Although cold storage is the current method for the preservation of hearts during the ex vivo transport interval, its disadvantages are highlighted in hearts from the extended criteria donor. In contrast, transport of high-risk hearts using hypothermic machine perfusion provides continuous support of aerobic metabolism and ongoing washout of metabolic byproducts. Perhaps more importantly, monitoring the organ's response to this intervention provides insight into the viability of a heart initially deemed as extended criteria. Ex vivo perfusion introduces challenges, such as ensuring homogeneous tissue perfusion and avoiding myocardial oedema. Though numerous groups have experimented with this technology, the best perfusate and perfusion parameters needed to achieve optimal results remain unclear. ${ }^{37}$

\section{Advances in surgical techniques}

Heart transplantation may be done with an orthotopic technique, with the donor heart implanted in the mediastinum in place of the native heart or heterotopic techniques with the donor heart implanted beside the native heart in parallel with it. This was done to militate against the risk of early graft failure which was high in the early era of heart transplantation. Heterotopic transplantation is rarely done today. There are two indications for heterotopic heart transplantation; patients with elevated pulmonary hypertension in whom the donor right ventricle would be unable to tolerate the increased after load; and significant size mismatch (donor/recipient weight ratio $>75 \%$ ), especially seen in pediatric patients and patients with irreversible elevation in pulmonary hypertension. The biatrial technique for orthotopic heart transplantation was first performed in a dog model by Lower and Shumway. ${ }^{38}$ Preservation was provided by the use of topical hypothermia induced by immersion of the graft in iced saline. ${ }^{3,38}$ Sievers et al., ${ }^{39}$ described a variation of the orthotopic procedure termed the bicaval technique where the donor right atrium is attached directly to the inferior and superior vena cava and the left atrial anastomoses is done as a cuff. Compared with biatrial approach, the bicaval approach results in less disruption of the atrial geometry, better right ventricular function, less tricuspid and mitral regurgitation, and less sinus node dysfunction. ${ }^{40}$

The operative steps are as follows: Through a mid-line sternotomy, the diseased heart is exposed. Following full systemic heparinisation, cannulation for cardiopulmonary bypass is accomplished with a straight aortic cannula, high in the ascending aorta. Venous cannulation is via a right angle or straight cannula directly into the superior vena cava or through the posterior right atrium into the superior vena cava. Inferior vena cava cannulation is accomplished through the posterior right atrium. Certain situations, such as recipient instability or difficult mediastinal dissection may need femoral cannulation for cardiopulmonary bypass. When the donor heart is close to the operating room the ascending aorta is cross-clamped and the diseased heart is excised. An incision is made in the right atrial appendage. This is extended inferiorly, anterior to the inferior vena cava cannula and towards the aorta root. The left atrium is entered through the superior limb of the fossa ovalis. The aorta and pulmonary arteries are transected just above their ventriculo-arterial valves. The interatrial septum is divided down to the coronary sinus, which demarcates the atrioventricular groove. Resection of the heart results in cuffs of left atrium, SVC, IVC, aorta, and pulmonary artery. Prior to implantation, the donor heart is inspected for a patent foramen ovale, which is closed by direct suture, and any other anomalies. Further myocardial protection in the form of $1000 \mathrm{ml}$ of cold blood cardioplegia may be given into the donor heart via its clamped aortic root. The implant procedure begins with anastomoses of donor and recipient left atria. Using a $4 / 0$ polypropylene monofilament suture, the anastomoses starts at the level of the left atrial appendage. Care is taken to align the interatrial septum. A transmitral vent is placed prior to completion of the left atrial anastomoses to vent the left ventricle and stop it from distending. Cardiac cooling may be augmented by running cold Ringer's solution through this vent during implantation. The next anastomoses are to the donor and recipient aorta. The donor aorta is left long so that there this is little tension on the anastomoses, and so it can be manipulated to allow inspection of the suture lines on completion of the transplant. This is done with continuous $4 / 0$ polypropylene monofilament suture. At this point the heart can be de-aired via a needle vent placed in the ascending aorta, and reperfusion begun with warm, leukocyte filtered blood at a carefully controlled pressure. Prior to release of the cross clamp, 500mg of methylprednisolone 
is given intravenously. The next anastomosis is the PA, this is done with continuous $5 / 0$ polypropylene. The pulmonary artery should be trimmed short. If the pulmonary artery is left too long, distortion can occur with reestablishment of normal right ventricular contraction. The final anastomoses are between the respective inferior and superior vena cavae, with continuous $5 / 0$ polypropylene, tied down initially to prevent purse stringing of these low pressure structures. When the patient has been rewarmed there follows at least $30 \mathrm{~min}$ of reperfusion. An isoprenaline infusion is commenced, pacing wires are placed on the surface of the heart and the patient is weaned from cardiopulmonary bypass. Protamine is administered to reverse the effects of heparin and haemostasis is performed, followed by closure. Despite the use of the bicaval technique, tricuspid regurgitation remains a problem early and late after heart transplantation. Adding a tricuspid annuloplasty ring to stabilize the annulus, to the transplant operation has been recently shown to decrease the incidence of tricuspid regurgitation and may even improve survival. ${ }^{41}$

\section{Conclusion}

The ultimate strength of heart transplantation is its excellent long term outcomes. It is the ideal solution for intractable end stage heart failure in eligible patients. The limiting factor is the limited availability of donor organs. An attempt to militate against this has to some extent been made by the development of ventricular assist devices. Device iterations with miniaturization and continuous flow have resulted in effective bridge to transplant solutions. The presence of an externalized drive line, however, exposes the VAD recipient to infections which may precipitate urgent listing for heart transplant in the bridge to transplant candidate and may limit the life span of the destination therapy candidate. Fully implantable driveline free systems will definitely enhance the utility of these systems in these settings. As our knowledge of molecular medicine increases, manipulation of key proteins implicated in the pathophysiology of heart failure such as SERCA2a may allow some recovery of the myocardium in patients with heart failure to the extent that transplantation may be deferred or the LVAD explanted. The other thrust to improve outcomes and meet need for organs is to find solutions to the low numbers of donors, which, as discussed, is further pressurized by the increased usage of DCD donors. Ex vivo perfusion systems are one answer to facilitate transport across large distances and allow the heart to be evaluated with a considerable reduction in ischemic time. Furthermore, systems in evolution in experimental models to allow reanimation of the DCD heart need to be translated into the clinical retrieval process. In situ perfusion of the DCD donor with ECMO is particularly attractive followed by ex vivo perfusion during transport.

\section{Acknowledgement}

None.

\section{Conflicts of interest}

Authors declare that there are no conflicts of interest.

\section{References}

1. Miller LW, Pagani FD, Russell SD, et al. Use of a continuous-flow device in patients awaiting heart transplantation. $N$ Engl $\mathrm{J} \mathrm{Med}$. 2007;357(9):885-896.

2. Slaughter MS, Rogers JG, Milano CA, et al. Advanced heart failure treated with continuous-flow left ventricular assist device. N Engl J Med. 2009;361(23):2241-2251.
3. Slaughter MS, Pagani FD, Rogers JG, et al. Clinical management of continuous-flow left ventricular assist devices in advanced heart failure. J Heart Lung Transplant. 2010;29(4 Suppl):S1-S39.

4. Levy WC, Mozaffarian D, Linker DT, et al. Can the Seattle heart failure model be used to risk-stratify heart failure patients for potential left ventricular assist device therapy? J Heart Lung Transplant. 2009;28(3):231-236.

5. Lietz K, Long JW, Kfoury AG, et al. Outcomes of left ventricular assist device implantation as destination therapy in the post-REMATCH era: Implications for patient selection. Circulation. 2007;116(5):497-505.

6. Teuteberg JJ, Ewald GA, Adamson RM, et al. Risk assessment for continuous flow left ventricular assist devices:Does the destination therapy risk score work? An analysis of over 1,000 patients. J Am Coll Cardiol. 2012;60(1):44-51.

7. Matthews JC, Koelling TM, Pagani FD, et al. The right ventricular failure risk score a pre-operative tool for assessing the risk of right ventricular failure in left ventricular assist device candidates. $\mathrm{J} \mathrm{Am} \mathrm{Coll} \mathrm{Cardiol.}$ 2008;51(22):2163-2172.

8. Fitzpatrick JR, Frederick JR, Hsu VM, et al. Risk score derived from preoperative data analysis predicts the need for biventricular mechanical circulatory support. J Heart Lung Transplant. 2008;27(12):1286-1292.

9. Stevenson LW, Pagani FD, Young JB, et al. INTERMACS profiles of advanced heart failure:The current picture. J Heart Lung Transplant. 2009;28(6):535-541.

10. Dardas T, Mokadam N, Pagani F, et al. Transplant registrants with implanted left ventricular assist devices have insufficient risk to justify elective organ procurement and transplantation network status 1 A time. $J$ Am Coll Cardiol. 2012;60(1):36-43.

11. John R, Long JW, Massey HT, et al. Outcomes of a multicenter trial of the Levitronix CentriMag ventricular assist system for short-term circulatory support. J Thorac Cardiovasc Surg. 2011;141(4):932-939.

12. Kiernan MS, Krishnamurthy B, Kapur NK. Percutaneous right ventricular assist via the internal jugular vein in cardiogenic shock complicating an acute inferior myocardial infarction. J Invasive Cardiol. 2010;22(2):E23-E26.

13. Granfeldt H, Hellgren L, Dellgren G, et al. Experience with the Impella recovery axial-flow system for acute heart failure at three cardiothoracic centers in Sweden. Scand Cardiovasc J. 2009;43(4):233-239.

14. Klotz S, Meyns B, Simon A, et al. Partial mechanical long-term support with the Circu Lite Synergy pump as bridge-to-transplant in congestive heart failure. Thorac Cardiovasc Surg. 2010;58(Suppl 2):S173-S178.

15. Seyfarth M, Sibbing D, Bauer I, et al. A randomized clinical trial to evaluate the safety and efficacy of a percutaneous left ventricular assist device versus intra-aortic balloon pumping for treatment of cardiogenic shock caused by myocardial infarction. J Am Coll Cardiol. 2008;52(19):1584-1588.

16. Cheng JM, den Uil CA, Hoeks SE, et al. Percutaneous left ventricular assist devices vs. intra-aortic balloon pump counterpulsation for treatment of cardiogenic shock:A meta-analysis of controlled trials. Eur Heart J. 2009;30(17):2102-2108.

17. Wieselthaler GM, O Driscoll G, Jansz P, et al. Initial clinical experience with a novel left ventricular assist device with a magnetically levitated rotor in a multi-institutional trial. J Heart Lung Transplant. 2010;29(11):1218-1225.

18. Waters B, Sample A, Smith J, et al. Toward total implantability using free-range resonant electrical energy delivery system:achieving untethered ventricular assist device operation over large distances. Cardiol Clin. 2011;29(4):609-625. 
19. Hall JL, Birks EJ, Grindle S, et al. Molecular signature of recovery following combination left ventricular assist device (LVAD) support and pharmacologic therapy. Eur Heart J. 2007;28(5):613-627.

20. Hall JL, Fermin DR, Birks EJ, et al. Clinical, molecular, and genomic changes in response to a left ventricular assist device. $\mathrm{J} \mathrm{Am} \mathrm{Coll} \mathrm{Cardiol}$. 2011;57(6):641-652.

21. Chaudhri B, Del Monte F, Hajjar RJ, et al. Interaction between increased SERCA2a activity and beta -adrenoceptor stimulation in adult rabbit myocytes. Am J Physiol Heart Circ Physiol. 2002;283(6):H2450-H2457.

22. Chaudhri BB, Del Monte F, Harding SE, et al. Gene transfer in cardiac myocytes. Surg Clin North Am. 2004;84(1):141-159.

23. Kawase Y, Ly HQ, Prunier F, et al. Reversal of cardiac dysfunction after long-term expression of SERCA2a by gene transfer in a preclinical model of heart failure. J Am Coll Cardiol. 2008;51(11):1112-1119.

24. http://clinicaltrials.gov/show/NCT00534703

25. Jaski BE, Jessup ML, Mancini DM, et al. Calcium up regulation by percutaneous administration of gene therapyin cardiac disease (CUPID Trial), a first-in-human phase 1/2 clinical trial. J Card Fail. 2009;15(3):171-181.

26. Birks EJ, George RS, Hedger M, et al. Reversal of severe heart failure with a continuous-flow left ventricular assist device and pharmacologic therapy:a prospective study. Circulation. 2011;123(4):381-390.

27. Butler CR, Jugdutt BI. The paradox of left ventricular assist device unloading and myocardial recovery in end-stage dilated cardiomyopathy:Implications for heart failure in the elderly. Heart Fail Rev. 2012;17(4-5):615-633.

28. Anastasiadis K, Antonitsis P, Argiriadou H, et al. Hybrid approach of ventricular assist device and autologous bone marrow stem cells implantation in end-stage ischemic heart failure enhances myocardial reperfusion. J Transl Med. 2011;19:9-12.

29. Copeland JG, Smith RG, Arabia FA, et al. Cardiac replacement with a total artificial heart as a bridge to transplantation. $N$ Engl J Med. 2004;351(9):859-867.

30. Copeland JG, Copeland H, Gustafson M, et al. Experience with more than 100 total artificial heart implants. J Thorac Cardiovasc Surg. 2012;143(3):727-734.
31. Christie JD, Edwards LB, Kucheryavaya AY, et al. The Registry of the International Society for Heart and Lung Transplantation:twenty seventh official adult lung and heart and lung transplant report-2010. J Heart Lung Transplant. 2010;29(10):1104-1118.

32. Ghodsizad A, Bordel V, Ungerer M, et al. Ex vivo coronary angiography of a donor heart in the organ care system. Heart Surg Forum. 2012;15(3):E161-E163.

33. Jamieson RW, Friend PJ. Organ reperfusion and preservation. Front Biosci. 2008;13:221-235.

34. Boucek MM, Mashburn C, Dunn SM, et al. Denver children's pediatric heart transplant team. pediatric heart transplantation after declaration of cardio circulatory death. $N$ Engl J Med. 2008;359(7):709-714.

35. Southerland KW, Castleberry AW, Williams JB, et al. Impact of donor cardiac arrest on heart transplantation. Surgery. 2013;154(2):312-319.

36. Mownah OA, Khurram MA, Ray C, et al. Development of an ex vivo technique to achieve reanimation of hearts sourced from a porcine donation after circulatory death model. J Surg Res. 2014;189(2):326334.

37. Longnus SL, Mathys V, Dornbierer M, et al. Heart transplantation with donation after circulatory determination of death. Nat Rev Cardiol. 2014;1(6):354-363.

38. Lower RR, Shumway NE. Studies on orthotropic homotransplantations of the canine heart. Surg Forum. 1960;11:18-19.

39. Sievers HH, Weyand M, Kraatz EG,et al. An alternative technique for orthotopic cardiac transplantation, with preservation of the normal anatomy of the right atrium. Thorac Cardiovasc Surg. 1991;39(2):70-72.

40. Schnoor M, Schafer T, Luhmann D, et al. Bicaval versusstandard technique in orthotopic heart transplantation:asystematic review and meta-analysis. J Thorac Cardiovasc Surg. 2007;134(5):1322-1331.

41. Jeevanandam V, Russell H, Mather P, et al. Donor tricuspid annuloplasty during orthotopic heart transplantation:long-term results of a prospective controlled study. Ann Thorac Surg. 2006;82(6):2089-2095. 